\title{
Nuclear Power Plant Operator Reliability Research Based on Fuzzy Math
}

\author{
Fang Xiang, Zhou Yangping, and Li Fu \\ Tsinghua University, Beijing 100084, China \\ Correspondence should be addressed to Fang Xiang, fxdirection@sina.com \\ Received 5 May 2011; Accepted 5 September 2011 \\ Academic Editor: Anis Bousbia Salah
}

Copyright () 2011 Fang Xiang et al. This is an open access article distributed under the Creative Commons Attribution License, which permits unrestricted use, distribution, and reproduction in any medium, provided the original work is properly cited.

This paper makes use of the concept and theory of fuzzy number in fuzzy mathematics, to research for the response time of operator in accident of Chinese nuclear power plant. Through the quantitative analysis for the performance shape factors (PSFs) which influence the response time of operators, the formula of the operator response time is obtained based on the possibilistic fuzzy linear regression model which is used for the first time in this kind of research. The research result shows that the correct research method can be achieved through the analysis of the information from a small sample. This method breaks through the traditional research method and can be used not only for the reference to the safe operation of nuclear power plant, but also in other areas.

\section{Introduction}

The reliability research of nuclear power plant (NPP) operators is focused on the non-response probability of NPP operators under the restrained time. The response time to the accidents is very important. It can be influenced by the performance shape factors (PSFs). Since PSFs include many factors and uncertainties, it is very difficult to be processed quantitatively. Based on the probability distribution of the response time on human cognitive reliability (HCR) model, in order to solve the uncertainty problem on the nonresponse probability, the response time is supposed to be a singlepoint value. This is the traditional research method of HCR model. However, because the uncertainty of the median time to the accidents on HCR model can be influenced by PSF, it is not completed to regard the response time as only a simplepoint value. In another word, the response time should be a range or an uncertain number.

The paper makes use of the related method of fuzzy mathematics and does the research of the NPP operator response time distribution related to PSF. The method describes the linear relations between the response time and PSF. The relations can be described by the fuzzy definition of PSF that can set up a linear fuzzy system equation and makes use of fuzzy linear regression to estimate the related fuzzy parameters. Furthermore, the possibilistic distribution function of the response time to the accidents of NPP operators can be obtained.

\section{Analysis of Possibilistic Linear Regression Model}

A linear system model whose parameters are defined by possibility distributions, not defined by probability functions, is called a possibilistic linear regression system model. Here, a possibility distribution is represented by a fuzzy number that has a membership function to surround its central value.

A possibilistic linear system can describe the system model that is influenced by many complicated and uncertain factors [1]

$$
Y=A_{1} x_{1}+\cdots+A_{n} x_{n}=A x,
$$

where $A_{i}=\left(\alpha_{i}, c_{i}\right)_{L}$ is a coefficient which is described by a fuzzy number, and $x_{i}$ is a real number that can influence system feature.

The fuzzy output $Y$ can be represented as

$$
Y=(\alpha x, c|x|)_{L}
$$


where $\alpha=\left(\alpha_{1}, \ldots, \alpha_{n}\right), c=\left(c_{1}, \ldots, c_{n}\right)$, and $|x|=$ $\left(\left|x_{1}\right|, \ldots,\left|x_{n}\right|\right)^{t}$.

Based on formula (1) and formula (2), a possibilistic linear system can be symbolically rewritten as

$$
\left(\alpha_{1}, c_{1}\right)_{L} x_{1}+\cdots+\left(\alpha_{n}, c_{n}\right)_{L} x_{n}=(\alpha x, c|x|)_{L} .
$$

In formula (1), with a degree $0 \leq h<1$, denoted as $A_{1} \supseteq_{h} A_{2}$, it is defined by $\left[A_{1}\right]_{h} \supset\left[A_{2}\right]_{h}$, equivalent to $[1]$

$$
\begin{aligned}
& \alpha_{1} \leq \alpha_{2}+\left|L^{-1}(h)\right|\left(c_{1}-c_{2}\right), \\
& \alpha_{1} \geq \alpha_{2}-\left|L^{-1}(h)\right|\left(c_{1}-c_{2}\right) .
\end{aligned}
$$

If $\left[A_{1}\right]_{h} \supset\left[A_{2}\right]_{h}$, for $h \in(0,1)$, we have

$$
\left[A_{1}\right]_{h^{\prime}} \supset\left[A_{2}\right]_{h^{\prime}} \quad \forall h^{\prime} \leq h .
$$

For the estimation of the parameters in the possibilistic linear fuzzy regression model, if there is a model for producing an upper set covering the fuzzy observed value, a lower set covered by the observed value, or a set intersected with the observed value, it could give estimates of the fuzzy parameters.

The given data are represented as $\left(Y_{i}, x_{i}\right), i=1, \ldots, N$, where $Y_{i}=\left(y_{i}, e_{i}\right)_{L}$ is a fuzzy observed value and $x_{i}=$ $\left(x_{i 1}, \ldots, x_{i n}\right)$ is a vector of explanatory variables for the $i$ th sample.

The possibilistic linear distribution can be described by fuzzy number $A$. The basic concept for formulating three possibilistic linear regressions is to use the mutual relation between the observed and estimated intervals which are obtained by $h$-level sets of the observed and the estimated fuzzy numbers, respectively.

Our idea is to obtain the fuzzy parameters $A_{i}, \underline{A}_{i}$, and $\hat{A}_{i}$, $i=1, \ldots, N$,

$$
\begin{gathered}
Y_{i} \subseteq_{h} \overline{Y_{i}}=\bar{A}_{1} x_{i 1}+\cdots+\bar{A}_{n} x_{i n}, \\
Y_{i} \supseteq_{h} \underline{Y}_{i}=\underline{A}_{1} x_{i 1}+\cdots+\underline{A}_{n} x_{i n}, \\
{\left[Y_{i}\right]_{h} \cap\left[\hat{Y}_{i}=\hat{A}_{1} x_{i 1}+\cdots+\hat{A}_{n} x_{i n}\right]_{h} \neq \varnothing .}
\end{gathered}
$$

The formulations derived from formula (6)-(8) are called Min problem, Max problem, and Conjunction problem, respectively.

The objective that we do the research on fuzzy numbers is that fuzzy numbers can describe not only a sample point but also a relevant range. The method can be used in reliability cognitive model of NPP operators. The individual NPP operator cognitive model is relevant to the related fuzzy number.

The mutual relations given by formula (6)-(8) are illustrated in Figure 1.

Min problem is

$$
\operatorname{Min}_{\overline{A_{j}}=\left(\overline{\alpha_{j}}, \overline{c_{j}}\right)_{L}} \bar{J}(\bar{c})=\sum \bar{c}\left|x_{i}\right|
$$

where $\bar{J}(\bar{c})$ is the fuzzy extent of fuzzy linear regression model.

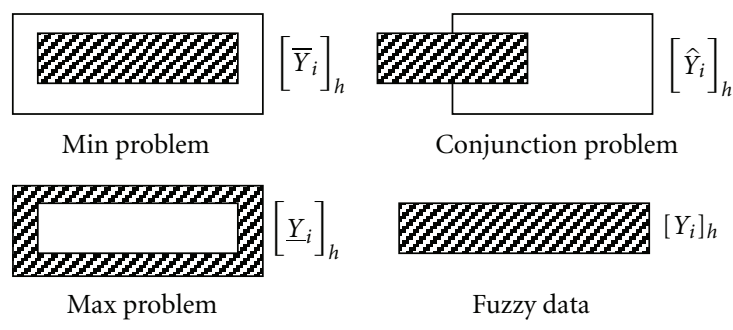

Figure 1: The concepts of three problems.

Subject to formula (6), $\bar{c}\left|x_{i}\right|$ is the spread of estimated fuzzy output $\overline{Y_{i}}$. This problem can be interpreted as obtaining the smallest spread of the fuzzy extent or obtaining the smallest spread of $\sum \bar{Y}_{i}$ such that $\bar{Y}_{i} \supseteq_{h} Y_{i}$. Using formulas (3) and (4), Min problem can be reduced to the following linear programming:

$$
\begin{aligned}
& \operatorname{Min}_{\bar{\alpha}, \bar{c}} \sum \bar{c}\left|x_{i}\right|=\bar{J}(\bar{c}), \\
& y_{i}+\left|L^{-1}(h)\right| e_{i} \leq \bar{\alpha} x_{i}+\left|L^{-1}(h)\right| \bar{c}\left|x_{i}\right|, \\
& y_{i}-\left|L^{-1}(h)\right| e_{i} \geq \bar{\alpha} x_{i}-\left|L^{-1}(h)\right| \bar{c}\left|x_{i}\right|, \\
& \bar{c} \geq 0, \quad i=1, \ldots, N .
\end{aligned}
$$

If fuzzy outputs are reduced to nonfuzzy outputs, the Conjunction problem is equivalent to the Min problem although the Max problem does not exist [2].

Among the mutual relations of the Min, Max, and Conjunction problems, the Min problem has the widest contents.

For instance, if there is an upper fuzzy set $Y^{U}=$ $\left(m^{U}, \delta^{U}\right)_{L}$, covering the fuzzy observed value $Y_{j}=\left(m_{j}, \delta_{j}\right)_{L}$ for $0 \leq \alpha<1$, based on formula (4), it is reduced to

$$
\begin{aligned}
& m^{U} \geq m_{j}-\left|L^{-1}(\alpha)\right| \times\left(\delta^{U}-\delta_{j}\right), \\
& m^{U} \leq m_{j}+\left|L^{-1}(\alpha)\right| \times\left(\delta^{U}-\delta_{j}\right) .
\end{aligned}
$$

If the values for $m^{U}$ and $\delta^{U}$ exist while minimizing $\delta^{U}$ in the above equations, those values can be used as estimates for the upper fuzzy set. This problem can be formulated as a linear programming (LP) problem. The possibilistic fuzzy linear regression model supplies the specific quantitative research method to the complicated system influenced by varied factors. The paper makes use of Min problem analysis method to do researches about the accidents response time of NPP operators.

Among three kinds of fuzzy number problems, Min problem method can be selected to do application in NPP operator reliability cognitive research. The reliability cognitive model of NPP operator can be described by using the widest range fuzzy number. 


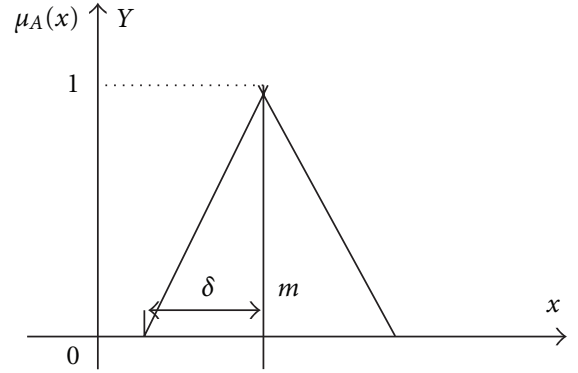

Figure 2: Symmetrical fuzzy number.

\section{Application to the Research of Cognitive Model of NPP Operators: Response Time Estimations}

The cognitive response time of NPP operator can be affected by varied PSF such as psychology, stress, procedure, cognitive complexity, and environments. The response time can be described by mathematics equations. The possibilistic linear regression model supplies PSF quantitative method to the complicated problem such as NPP operator accident response time.

Based on the data of the experimental accident response time of Chinese NPP operators, it is assumed that the response times can be represented by the fuzzy numbers which have symmetrical possibility distributions around the observed values. That is, each observed value becomes a center point having the possibility of 1 , and the possibility distribution is symmetrically spread from the center point based on $\delta$ value. The wider the spread of possibility distribution, the more fuzziness the response time has. Figure 2 shows the symmetrical fuzzy number. The symmetrical fuzzy number $Y$ is represented as $Y=(m, \delta)_{L}$, where $m$ is the center value, $\delta$ is the spread of fuzzy number, and the membership function is $\mu_{A}(x)$. In this paper, the spread $\delta$ is assumed to be 10 seconds for the response times based on the experimental data and experts suggestions.

In this paper, we only use one accident: the loss of coolant accident (LOCA) as the research example so as to decrease the data volume. The response time of the LOCA accident on the Chinese NPP operators can be studied through the possibilistic linear fuzzy regression model. Table 1 shows the biographical data and the corresponding experimental observed response times of Chinese operators ( 24 crew in 8 groups).

In Table 1, the basic response time value of LOCA is the NPP simulator experiment result.

The related data represented by fuzzy numbers of LOCA accident of 24 Chinese NPP operators in 8 groups are shown in Table 2.

In Table 2, the accident response time, age, experience inside and outside control room, and education background are the average value of Chinese NPP operators.

Since all the 24 of the crew have no experience inside control room, thus the relevant factor can be omitted. The possibilistic linear model for the response time $Y_{j}$ is assumed to be as follows:

$$
Y_{j}=A_{0}+A_{1} X_{1 j}+A_{2} X_{2 j}+A_{3} X_{3 j},
$$

where $X_{1 j}, X_{2 j}$, and $X_{3 j}$ denote age, experience outside a control room, and education background for the $j$ th crew group, respectively. When $Y_{j}$, is the fuzzy observed value denoted by $Y_{j}=\left(Y_{j}, \delta_{j}\right)_{L}$ with respect to the $j$ th input variable $X_{j}=(X 1, \ldots, X 3)_{j}$ and $A_{i}$ are fuzzy numbers denoted by $\left(m_{i}, \delta_{i}\right)_{L}, i=0, \ldots, 3, A_{0}$ denotes the accident response time represented by fuzzy parameter of NPP operators, and $A_{1}, A_{2}$, and $A_{3}$ denote age, experience outside a control room, and education represented by fuzzy parameters. Formula (12) can be written as

$$
\begin{aligned}
\left(y_{j}, \delta_{j}\right)_{L}= & \left(m_{0}, \delta_{0}\right)_{L}+\left(m_{1}, \delta_{1}\right)_{L} X_{1 j} \\
& +\left(m_{2}, \delta_{2}\right)_{L} X_{2 j}+\left(m_{3}, \delta_{3}\right)_{L} X_{3 j} \\
& j=1, \ldots, 8
\end{aligned}
$$

where $j$ represents the $j$ th observation group.

When using the Min problem, referring to formulas (11), LP problem can be formulated as follows:

$$
\begin{aligned}
& \min \sum_{j=1}^{8}\left(\delta_{0}^{U}, \delta_{1}^{U}, \ldots, \delta_{3}^{U}\right) \times\left(1, X_{1}, \ldots, X_{3}\right)_{j}^{T} \\
& \text { s.t. } \quad y_{j}+\left|L^{-1}(\alpha)\right| \times \delta_{j} \\
& \leq\left(m_{0}^{U}, m_{1}^{U}, \ldots, m_{3}^{U}\right) \times\left(1, X_{1}, \ldots, X_{3}\right)_{j}^{T} \\
&+\left|L^{-1}(\alpha)\right| \times\left(\delta_{0}^{U}, \delta_{1}^{U}, \ldots, \delta_{3}^{U}\right) \\
& \times\left(1, X_{1}, \ldots, X_{3}\right)_{j}^{T}, \\
& y_{j}-\left|L^{-1}(\alpha)\right| \times \delta_{j} \\
& \geq\left(m_{0}^{U}, m_{1}^{U}, \ldots, m_{3}^{U}\right) \times\left(1, X_{1}, \ldots, X_{3}\right)_{j}^{T} \\
&-\left|L^{-1}(\alpha)\right| \times\left(\delta_{0}^{U}, \delta_{1}^{U}, \ldots, \delta_{3}^{U}\right) \\
& \times\left(1, X_{1}, \ldots, X_{3}\right)_{j}^{T}, \quad \delta_{0}^{U}, \delta_{1}^{U}, \ldots, \delta_{3}^{U} \geq 0, \quad j=1, \ldots, 8
\end{aligned}
$$

where ()$^{T}$ represents matrix transform.

Through Table 2 and s.t. formula (14), we can get the results as follows:

$$
\begin{gathered}
\left(\delta_{0}, \delta_{1}, \delta_{2}, \delta_{3}\right)=(10,0,0,0) \\
\left(m_{0}, m_{1}, m_{2}, m_{3}\right)=(1417.2,-121.8,82.27,95.45) .
\end{gathered}
$$

The optimal solutions for $A_{0}, \ldots, A_{3}$ are obtained as

$$
\begin{aligned}
& A_{0}=(1417.2,10)_{L}, \\
& A_{1}=(-121.8,0)_{L}, \\
& A_{2}=(82.27,0)_{L}, \\
& A_{3}=(95.45,0)_{L} .
\end{aligned}
$$


TABLE 1: LOCA accident experimental result and the Chinese NPP operators data.

\begin{tabular}{|c|c|c|c|c|c|}
\hline Crew & Group & Age (year) & $\begin{array}{c}\text { Experience inside/outside a } \\
\text { control room (year) }\end{array}$ & Education (year) & $\begin{array}{l}\text { LOCA accident response time } \\
(\mathrm{sec})\end{array}$ \\
\hline 1 & 1 & 25 & $0 / 4$ & 14 & 10 \\
\hline 2 & 1 & 24.84 & $0 / 3$ & 15 & 26 \\
\hline 3 & 1 & 27 & $0 / 6$ & 15 & 18 \\
\hline 4 & 2 & 27.5 & $0 / 4$ & 17 & 48 \\
\hline 5 & 2 & 25 & $0 / 4$ & 14 & 14 \\
\hline 6 & 2 & 25.42 & $0 / 3$ & 16 & 82 \\
\hline 7 & 3 & 24.33 & $0 / 3$ & 15 & 129 \\
\hline 8 & 3 & 25 & $0 / 3$ & 16 & 82 \\
\hline 9 & 3 & 23.67 & $0 / 4$ & 14 & 47 \\
\hline 10 & 4 & 25.33 & $0 / 4$ & 14 & 33 \\
\hline 11 & 4 & 25 & $0 / 3$ & 15 & 19 \\
\hline 12 & 4 & 24.67 & $0 / 3$ & 15 & 26 \\
\hline 13 & 5 & 23.5 & $0 / 2$ & 14 & 88 \\
\hline 14 & 5 & 25 & $0 / 3$ & 14 & 10 \\
\hline 15 & 5 & 24.17 & $0 / 2$ & 15 & 50 \\
\hline 16 & 6 & 23.5 & $0 / 2$ & 14 & 42 \\
\hline 17 & 6 & 25.25 & $0 / 3$ & 15 & 60 \\
\hline 18 & 6 & 25.67 & $0 / 3$ & 15 & 60 \\
\hline 19 & 7 & 30 & $0 / 10$ & 15 & 49 \\
\hline 20 & 7 & 23.5 & $0 / 2$ & 15 & 126 \\
\hline 21 & 7 & 28.25 & $0 / 7$ & 15 & 19 \\
\hline 22 & 8 & 25.33 & $0 / 2$ & 15 & 76 \\
\hline 23 & 8 & 25 & $0 / 5$ & 14 & 41 \\
\hline 24 & 8 & 26.33 & $0 / 3$ & 15 & 59 \\
\hline
\end{tabular}

TABle 2: Fuzzy parameters of LOCA accident of Chinese NPP operators.

\begin{tabular}{lccc}
\hline $\begin{array}{l}\text { Response time }(\mathrm{sec}) \\
Y_{j}=\left(m_{j}, \delta_{j}\right)_{L}\end{array}$ & Age (year) & $\begin{array}{c}\text { Experience } \\
\text { inside/outside } \\
\text { a control room } \\
\text { (year) }\end{array}$ \\
\hline$(18,10)_{L}$ & 25.6 & $0 / 4.33$ & 14.67 \\
$(48,10)_{L}$ & 26.0 & $0 / 3.67$ & 15.67 \\
$(86,10)_{L}$ & 24.3 & $0 / 3.33$ & 15.0 \\
$(26,10)_{L}$ & 25.0 & $0 / 3.33$ & 14.67 \\
$(49.3,10)_{L}$ & 24.2 & $0 / 2.33$ & 14.33 \\
$(54,10)_{L}$ & 24.8 & $0 / 2.67$ & 14.67 \\
$(64.7,10)_{L}$ & 27.3 & $0 / 6.33$ & 15.0 \\
$(58.7,10)_{L}$ & 25.6 & $0 / 3.33$ & 14.67 \\
\hline
\end{tabular}

Thus, the possibilistic linear regression model for the response time $Y$ is

$$
\begin{aligned}
Y= & (1417.2,10)_{L}+(-121.8,0)_{L} X_{1} \\
& +(82.27,0)_{L} X_{2}+(95.45,0)_{L} X_{3},
\end{aligned}
$$

where $X_{1}, X_{2}$, and $X_{3}$ denote the variable of age, experience outside a control room, and education, respectively.
Based on formula (16), $A_{0}$ is a positive value, and the accident response time of NPP operator has a constant basic fuzzy number. The function of PSF can be realized through $A_{1}, A_{2}$, and $A_{3}$. The coefficient of the age of the operators $A_{1}$ is negative, it makes clear that the older the age is, the more possibility the rapid response based on experience has. The feedback is negative to the response time. Both the coefficient of the experience outside a control room of operators $A_{2}$ and the coefficient of the education $A_{3}$ are positive. It shows that rich working experience and good education can improve the synthetic quality and the judge ability.

\section{The Comparison between Fuzzy Parameters and Experimental Data}

In order to verify the fitting result of the model, the experimental data of the response time of NPP operators can be used for estimation. Using formula (17), the upper and lower fuzzy response time of each group can be estimated. Comparing the experimental data of the response time to the fuzzy estimated value of the response time, if the estimated intervals are falling within or being intersected with the probabilistic limits, the possibilistic fuzzy regression model can be regarded as a good model for estimating response times by using PSF. 


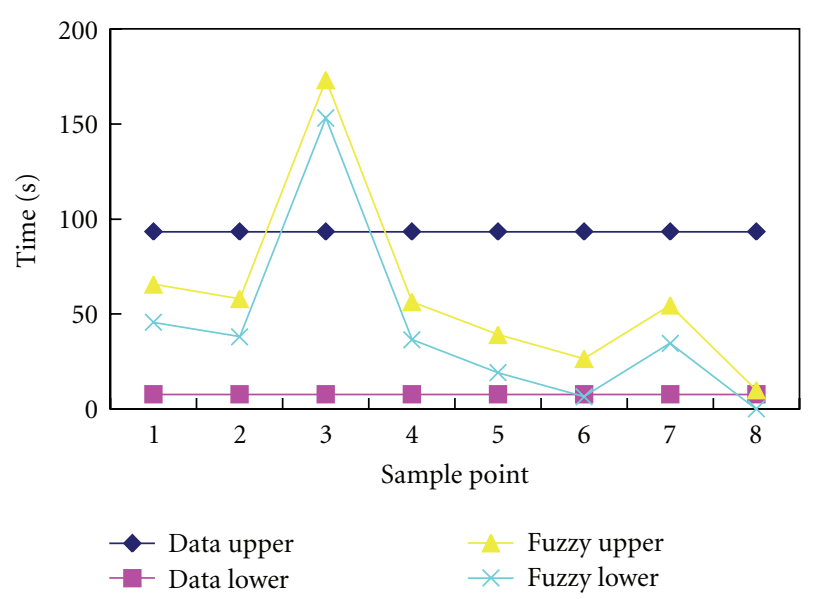

Figure 3: Fuzzy estimates versus data bound.

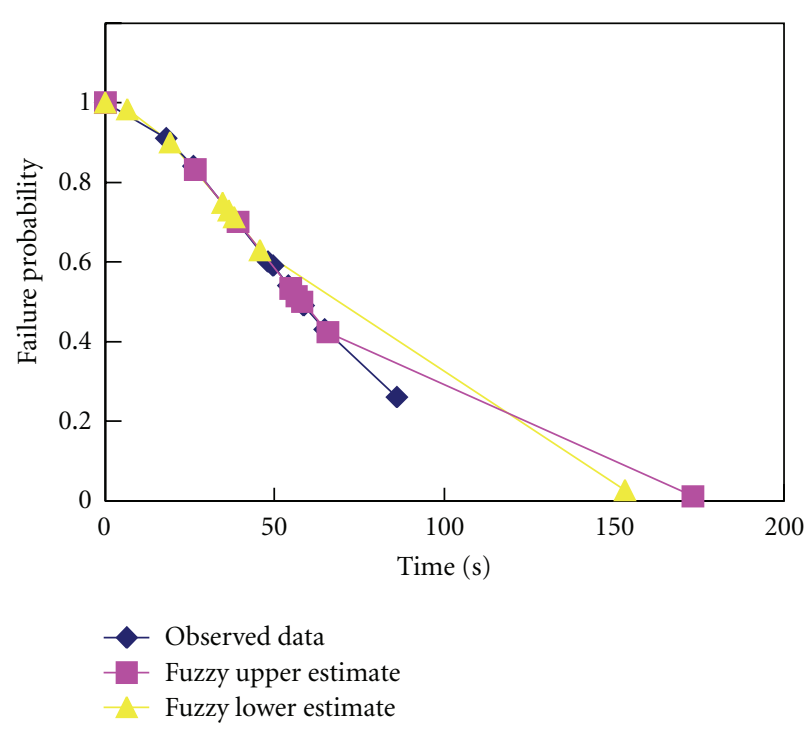

FIGURE 4: Failure probability curves.

Figure 3 shows the plots of the fuzzy upper and lower estimate intervals and the probabilistic upper and lower limits based on formula (13) and (17), respectively. From Figure 3, we can see that most of the fuzzy estimated intervals are falling within or being intersected by the $\pm 2 \sigma$ limits. It is believed that the fuzzy regression model can suggest some clues for the relation between response times and PSF based on small samples.

We also can determine if the failure probability plots of the fuzzy estimated upper and lower values were close to the failure probability based on experimental data in Table 2. The curves are shown in Figure 4 . Figure 4 shows that the failure probability curve from the observed data is between the failure probability curves from fuzzy estimated lower and upper values. The result can suggest an uncertainty bound of failure probability for a given response time. The fuzzy upper and lower limits have been formulated while coming as close to the observed value as possible. The intent of the comparison to the widths of the uncertainty of the failure probabilities at the given times is that if a model can give

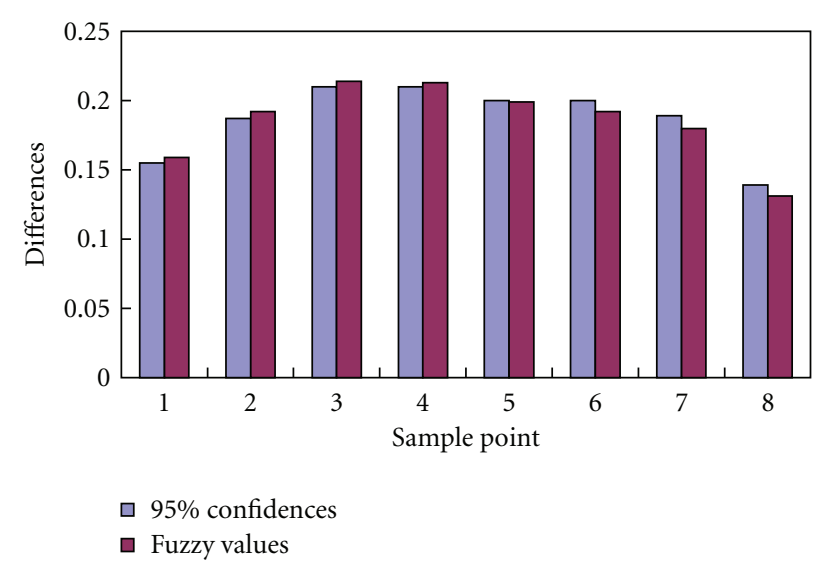

FIGURE 5: Comparison of the differences between upper and lower limits.

narrower widths of the uncertainty, the model will be able to ascertain the failure probability with more accuracy.

Figure 5 shows the comparison of the failure probability differences between the upper and lower 95\% confidences as well as the fuzzy upper and lower limits at the given times. The fact can be ascertained in Figure 5, where the trend of the differences between the upper and lower 95\% limits implies that the uncertainty of the failure probability is decreasing very gradually rather than quickly after around the mean time. Thus, if a given time is beyond the mean time, the lower failure probability for the given time becomes too small compared to the upper value. It may lead the HRA analyst to take the conservative direction when deciding human error probability. Contrarily, the differences from the fuzzy limits are decreasing sharply after the mean time, which indicates that the lower is close to the upper. This may result in avoiding conservative decision making. Even though it was impossible to compare the observed response times with the estimated values, we speculate that the possibilistic fuzzy regression model explains quite well the relation between response time and PSF in human reliability analysis.

The possibilistic linear fuzzy regression model supplies the research method arriving at target rapidly under the small samples. This will do good to the research of the reliability of Chinese NPP operators.

\section{Concluding Remarks}

Doing research on operator failure probabilities based on the fuzzy regression model in HRA can provide a well-fitted relationship between PSF (e.g., age, experience outside a control room, and education) and operator response time. Although because of different experiment conditions we can obtain different results, it is believed that the fuzzy regression model can play an important role, providing analysts with approximated failure probabilities. Therefore, the research method of fuzzy mathematics is a very useful tool to study NPP operator cognitive model.

The paper for the first time utilizes possibilistic linear fuzzy regression model to do the theory analysis and the quantitative calculation under small samples on the accident 
response time of Chinese NPP operators. The results are satisfied. The research method not only has important significance to the further research on Chinese NPP operators but also supplies good reference for the applications in other areas in the future.

\section{References}

[1] H. Tanaka, "Fuzzy data analysis by possibilistic linear models," Fuzzy Sets and Systems, vol. 24, no. 3, pp. 363-375, 1987.

[2] H. Tanaka, I. Hayashi, and J. Watada, "Possibilistic linear regression analysis for fuzzy data," European Journal of Operational Research, vol. 40, no. 3, pp. 389-396, 1989. 

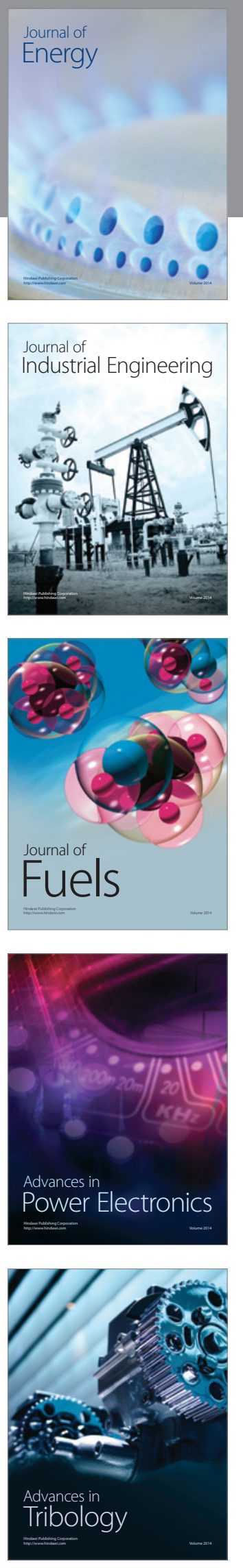
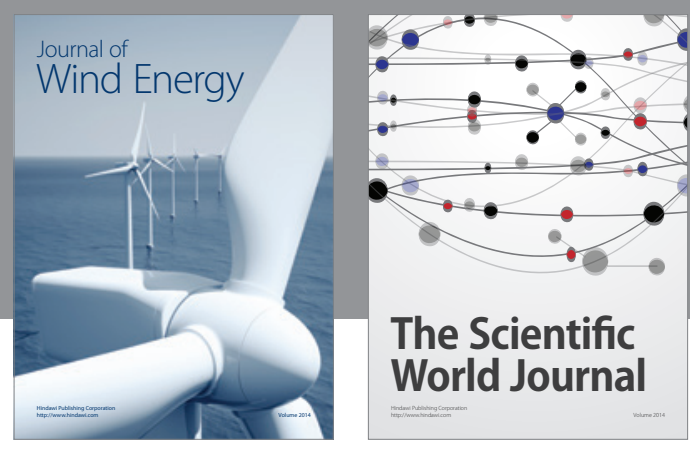

The Scientific World Journal

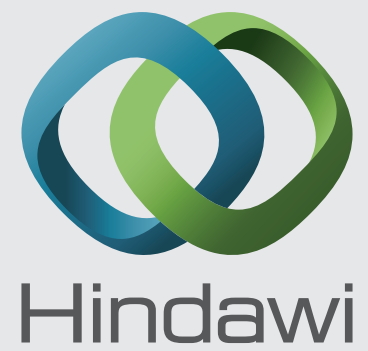

Submit your manuscripts at http://www.hindawi.com
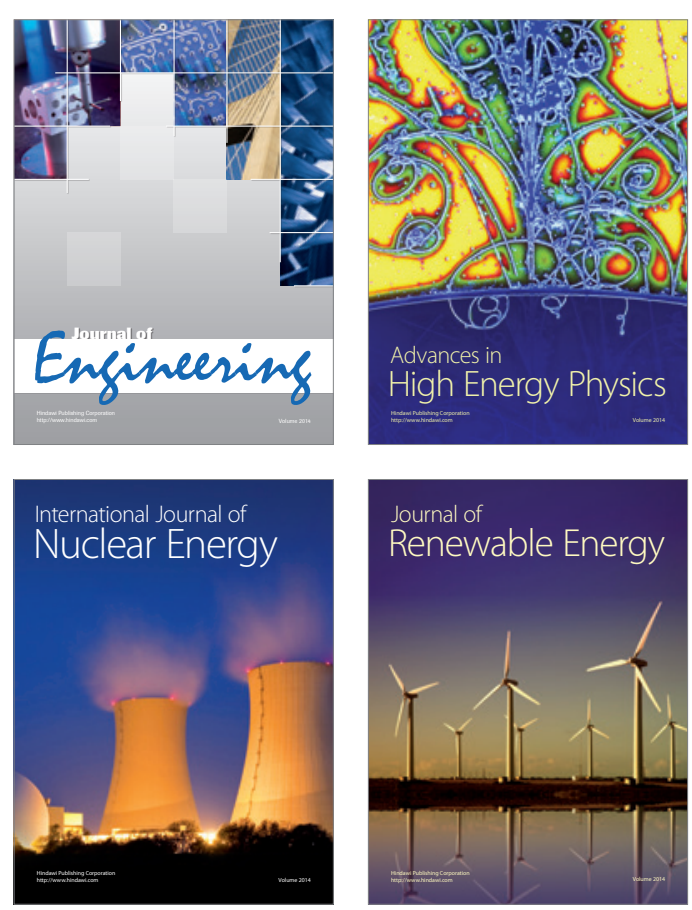

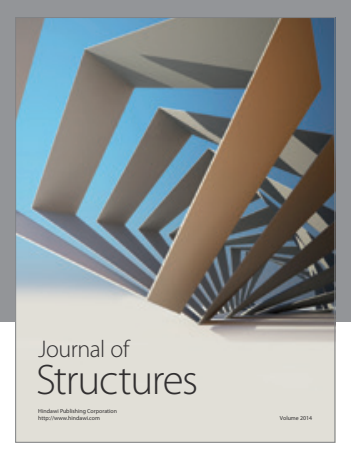

Rotating
Mechinery
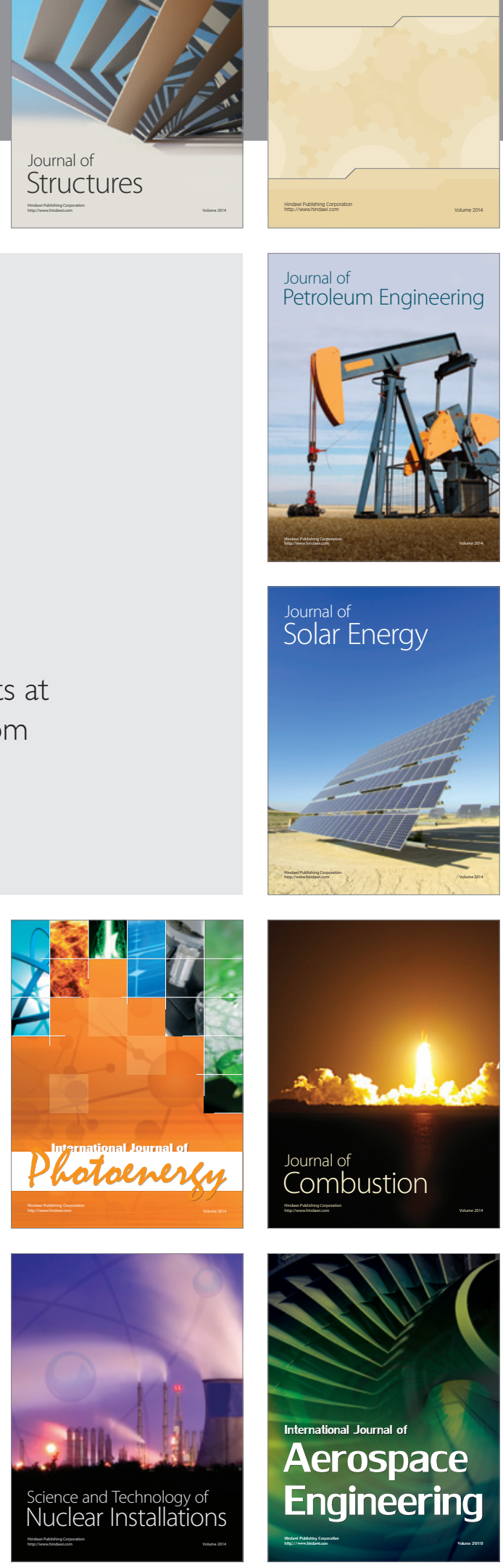